\title{
The Method of Dosed Directional Solidification (DDS) for Casting Turbine Blades
}

\author{
Alexander Stepanovich Verin \\ All-Russian Institute of Aircraft Materials, Moscow, Russia \\ Email address: \\ verin1937@list.ru

\section{To cite this article:} \\ Alexander Stepanovich Verin. The Method of Dosed Directional Solidification (DDS) for Casting Turbine Blades. Engineering Science. \\ Vol. 5, No. 1, 2020, pp. 1-4. doi: 10.11648/j.es.20200501.11
}

Received: January 3, 2020; Accepted: February 17, 2020; Published: May 15, 2020

\begin{abstract}
In this paper it is study a problem of multigrid schemes in two space dimensions and differential operator formula of Laplace and Lagrang for numerical tests, including a composition parameters of microstructures obtained by the DDS method. This technological possibilities of directed crystallization are broad and make it possible to manufacture castings from high- temperature alloys with various sets of properties. The present work is devoted to the possibilities of intensifying directed crystallization of the $\mathrm{Ni}_{3} \mathrm{Al}$ intermetallic compound by improving of feeding the growth casting, which makes it possible to control the process of structure formation and thus change the properties of material. Directed crystallization of the intermetallic compound is a very complex and unsteady process. This seems to be connected not only with that aluminum segregates in the mother solution that feeds the growing casting but also with the scale factor, i. e., the height of the column of the mother melt and its mold. We can infer from these data that the crystallization conditions in fabricating castings by the developed method are in better equilibrium. These conditions are provided by the dosed feeding of the liquid metal into the casting zone. The alloy obtained by the suggested method has higher mechanical characteristics for temperature ranging from room temperature to $1200^{\circ} \mathrm{C}$ than metal melted with the use of directed crystallization by the conventional method (CDS).
\end{abstract}

Keywords: Intermetallid $\mathrm{Ni}_{3} \mathrm{Al}$, Structure, $100 \mathrm{~h}$. Strength, Model

\section{Introduction}

In finish years of the twentieth century there have been shown a scale of using the partial derivative in process solving of problem numerical tests base-on the abstract framework. The basically part of this problem inexact methods in different fields of numerical analysis. In each separate case, such conditions of convergence are affine invariant and in agreement with the theory. In this case may be right to remark that exists inexact (example Newton iterative methods), where an iterative method is used to approximate the solution of the linear system [1].

However, in certain cases there is simply no alternative method at all to predict the behavior of a nonlinear, example, non-stationery distribution of crystallize phases in solid-liquid area growing ingots. A geometrically complex shape of neighbouring elements in the mesh are joined with each other only at their common nodes at displacement fields of dynamical system. However, it is connect (or satisfied), probably, for solid systems using finite element method during (as example) approximation the microstructure with layers which are oriented with respect to meshes. For example, it is simulate the deformation of martensitic crystals. In our case it is to note that the displacement of a certain node is a linear function caused by the external forces. This load is affecting seemly to opposite grown ingot. In the time crystallization in the solid-liquid area we can generate the different conditions of growth which may predict the different microstructure. Changing the direction of dosed parts of mother melt into solid-liquid area of the growing ingot we are obtaining the dendrites form (Figure 1) or cellular structure (Figure 2). In this case it is regard the nodes as cells. Obviously, it is an iterative formulation of idea may be from cellular aspect. In our case the mesh can be presented of rectangular type with L-shaped domain. The more details and numerical results for our version of the boundary element method will be shown in next papers. In order to construct our iteration version we used a natural network based-on a cellular microstructure $\mathrm{Ni}-12 \mathrm{Al}\left(\mathrm{Ni}_{3} \mathrm{Al}\right)$ compound. In this paper we applied the interpolation formula of Lagrang for analysis and control of parameters cell and dendrites. 


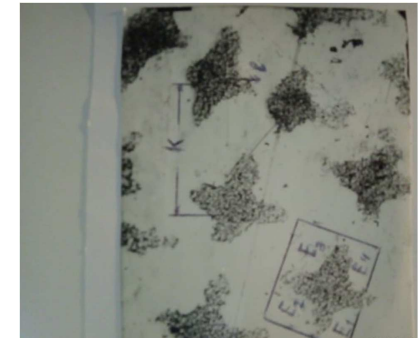

Figure 1. Dendritic microstructure of a casting obtained by dosed feeding of the liquid metal (DDS method) into ceramic mold in trasverse direction ( $x$ 100). $\mathrm{Ni}-12 \mathrm{Al}$ composition ( $\mathrm{Ni}_{3} \mathrm{Al}$ ).

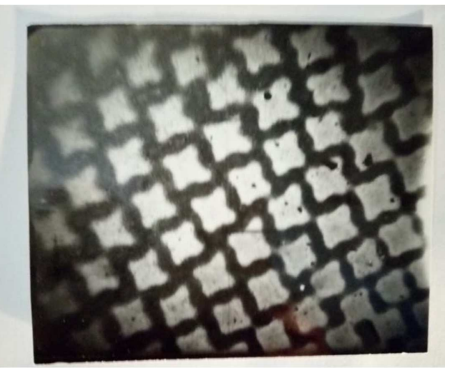

Figure 2. Cellular microstructure of a casting obtained by DDS method in trasverse direction (x100), $\mathrm{Ni}_{3} \mathrm{Al}$.

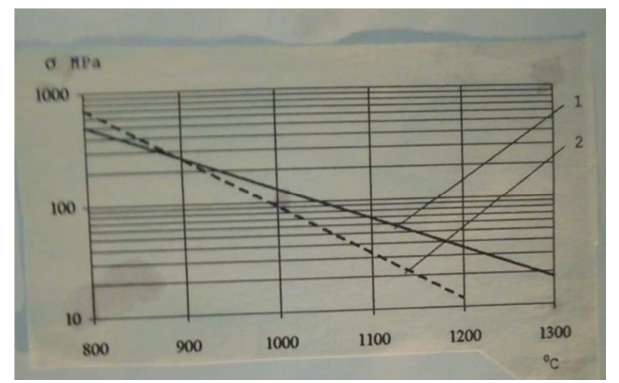

Figure 3. 100 hour long life. Where: $1-\mathrm{Ni}_{3} \mathrm{Al}$, 2- GS6-F (Ni, Co, W, Mo, Ti,........) Russian Ni superalloy.

\section{Experimental Part}

Changing the direction of dosed parts of mother metal in process directional solidification ingots and velocity growth we decreased the parameter k (Figure 1). In the result this we obtained different structural views and strength properties. A scheme of growth and transformation of dendrite axis (Figure 1) was done on base of microstructure on Ni-12Al. The figures 1 and 2 clearly demonstrate the improvement of the behavior of dendrites forms. This process reflects a connection between concentration of $\mathrm{Al}$ and velocity growth of the solid-liquid area of ingot. However, it is process to describe of mathematical very difficult. At the first step we used mapping technique which is based on equations Laplace, Lagrang and transformation a general domain $(\Omega)$ into the interior of a unite circle. We will not comment all mathematical indexes which we use in our graphic. It is a very comprehensive survey of the research prior 1981, example Mostin and Thompson, Sparis and et. al. P. Picard, et. al. 1998, John D. Hobby. 1993, Michael J. Mossinghoft. 1998, A. K. Lenstra, et. al. 1982. J. Hastad, et. al. L. Babai. 1986. Eric Grosse, at. al. 1994, A Leutbecher. 1989, K. R.
Jackson. 1996, J. M. Martinez, et. al. 1995.

In our case construction of numerical mapping may be requires the solution of a non-linear integral equation. It is main disadvantage. It is using the interpolation formula of Lagrang we obtained polynomials of a degree 3 . For this aim the parameter a $\left(\mathrm{a}_{0}, \mathrm{a}_{1}, \mathrm{a}_{2}, \mathrm{a}_{3}\right)$ is taken in limit [0.2-0.8], [2.0-2.6], [3.6-4.2], [5.2$5.8]$ correspondently by step of the arithmetical progression 0.2 . The parameter $b\left(b_{0}, b_{1}, b_{2}, b_{3}\right)$ is taken in limit [11.8-12.8], [12.9-13.9], [14.2 -15.2], [15.2-16.2 [correspondently by step of the arithmetical progression 0.3 .

Let $\Omega$ be the unite square $[0,1]^{2}$. In our case an additional constraint in the mesh generation, that faces on $\partial \Omega$ are represented by element faces which allows to create $\mathrm{E}$ with minimal k (Figure 1) suppose that our mesh involves a natural segmentation of $\Omega \backslash \Omega_{\mathrm{k}}$ into sets $\Omega^{\mathrm{E}}$ associated to boundary faces $E \in \partial_{\mathrm{k}}$. In result solution of Lagrang equation we obtained next polynomial of view:

$$
\mathrm{Ax}^{3}-\mathrm{Bx}^{2}+\mathrm{Cx}-\mathrm{D}
$$

where $\mathrm{A}$ has value from 0.1 to 1 in each system of equations. The solution of such equations have been positive root, which was using into process numerical calculation based-on differential operator $h^{2} \nabla^{2}$. The first task in showing that function $\mathrm{N}_{\mathrm{x}}$ may be determined as partial derivates. Thus we might expect these polynomials with all its roots on the unite circle in the interval $[-1,1]$ to be good candidates for integration of one-dimensional domains using formula Laplace $(\Delta \mathrm{N}=0)$. Such as $\mathrm{k} \neq \ell$ (it is shown in [2]) we used next operator;

$$
6 \mathrm{~N}_{\mathrm{i}}=\mathrm{N}_{\mathrm{a}}+\mathrm{N}_{\mathrm{c}}+4 \mathrm{~N}_{\mathrm{b}}
$$

[2] where:

$\mathrm{N}_{\mathrm{i}}$ is partial derivates of function $\mathrm{N} . \mathrm{N}_{\mathrm{a}}, \mathrm{N}_{\mathrm{b}}, \mathrm{N}_{\mathrm{c}}$ are value of function $\mathrm{N}$. The function $\mathrm{N}$ is denoted concentration of $\mathrm{Al}$ in solid-liquid area. By a straightforward calculation, the following induction scheme was searched based-on a rectangular grid [3-5]. The results of calculation are shown in table.

Table 1. values for sequence of grid in numerical samples.

\begin{tabular}{lllllll}
\hline \multirow{2}{*}{ Point } & \multirow{2}{*}{$\begin{array}{l}\text { Initial } \\
\text { point }\end{array}$} & $\mathbf{1}$ & $\mathbf{2}$ & $\mathbf{6}$ & $\mathbf{7}$ & Al \% \\
\cline { 3 - 6 } & & & & & & \\
\hline 23 & 0.3556 & 0.8926 & 0.9791 & 0.9997 & 0.9999 & 12.9999 \\
22 & 0.3556 & 0.8446 & 0.9652 & 0.9996 & 0.9998 & 12.9998 \\
21 & 0.3556 & 0.8124 & 0.9612 & 0.9994 & 0.9995 & 12.9995 \\
20 & 0 & 0.8020 & 0.9506 & 0.9976 & 0.9969 & 12.9969 \\
17 & -3556 & 0.368 & 0.5675 & 0.6547 & 0.6544 & 12.6544 \\
16 & 0 & 0.6029 & 0.7599 & 0.9399 & 0.9402 & 12.9402 \\
15 & 0 & 0.7428 & 0.8573 & 0.9892 & 0.9895 & 12.9895 \\
14 & 0.3556 & 0.8855 & 0.9046 & 0.998 & 0.9981 & 12.9981 \\
11 & 0 & 0.6977 & 0.7442 & 0.9856 & 0.9849 & 12.9849 \\
10 & -0.3556 & 0.4359 & 0.7718 & 0.9174 & 0.8993 & 12.8993 \\
9 & -3556 & 0.3079 & 0.3698 & 0.4888 & 0.6089 & 12.6089 \\
8 & -3556 & 0.1299 & 0.1393 & 0.3512 & 0.3716 & 12.3716 \\
\hline
\end{tabular}

Remark: denote that do not show 3 iterations.

Such mapping greatly simplifies the boundary conditions and ensures that this includes the general polyhedral case of 
rectangular grids. It is found that a distance between second arms of the dendrites in direction [001] always constant and equal not more $0.02 \mathrm{~mm}$. This direction is taken for determining the parameter $\mathrm{k}$. However a distance between second arms of the dendrites in direction [010] not constant because $\mathrm{k} \neq \ell$. The first we give the elementary argument for using formula 2 and others follow by symmetry arguments. This lead us to integrating this expression along the path [100] where $\pm 1 \geq \mathrm{x} \geq 0, \mathrm{y}=\mathrm{o}$. However, some of the multigrid are only valid in the case of the Laplace condition $(\Delta \mathrm{N}=0)$ in center of the grid. For calculation of concentration of $\mathrm{Al}$ in solid-liquid area in the time of the crystallization of ingot we are taken a value of rational root equal 0.3556 which was obtained after solution of polynomial:

$$
\mathrm{P}_{\mathrm{x}}=\mathrm{x}^{3}-7 \mathrm{x}^{2}+19 \mathrm{x}-6
$$

Such polynomials have been obtained in each interval of interpolation.

\section{Results}

In order to show the analytics efficiency and the strength of the characteristic DDS repesentation by mathematic way, we developed a prototype system called numerical using partial derivatives. With this system we have performed the some experiments which were differenced from each other $\mathrm{k}$ parameter. The parameter $\mathrm{k}$ was varied from 0.1-0.2 mm (velocity crystallization $20 \mathrm{~mm} / \mathrm{min}$ with cooling mold in melt tin) to $0.8-1 \mathrm{~mm}$ (velocity crystallization $1 \mathrm{~mm} / \mathrm{min}$ with cooling mold on water-cooled crystallizator). It was found that the smaller parameter $\mathrm{k}$ the higher a strength of samples at $1200^{\circ} \mathrm{C}$. It is connection probably with those that concentration of $\mathrm{Al}$ in solid-liquid area of ingots has a constant value. For the case when $\mathrm{k}=0.2 \mathrm{~mm}$ the concentration of $\mathrm{Al}$ in solid-liquid area is shown in Table, Such system is said to be controllable if, by a suitable manipulation of dose metal inputs, the state can be made to behave in some expected way, and this, in a time inlet doses may be calculated as precisely as possible. This concept is analyzed for neutral differential system. A geometric dendrites interpretation in term of state configuration is given, based on the definition of criss-cross forms precipitations in (001) crystallographic plane. In the general case this system may be used only via some groups of the dendrites precipitations. Using this abstract framework we can change the sensitivity of dosed directional process to govern of properties of composition $\mathrm{Ni}$ $12 \mathrm{Al}\left(\mathrm{Ni}_{3} \mathrm{Al}\right)$. It will play a fundamental role in deriving reliable this alloy for using in construction of turbine blades for future aircraft engines [6-8].

The results of tests of plane specimens (Ni-12Al) for longterm strength compared with standard specimens base-on superalloy GS6-F in a temperature range of $800-1200^{\circ} \mathrm{C}$ $(100 \mathrm{~h})$ are presented in Figure 3. The specimens of GS6-F were obtained by conventional directional solidification method. Besides, the alloy (Ni-12Al) obtained by DDS method has higher mechanical characteristics in this a temperature range than those alloy with the use of conventional directed method. This result is confirmed by numerical experiments which also demonstrate that such a shift of the quantity points in the grid often improves the precision of a selection velocity crystallization of ingots. In order to quantity the displacement of the interpolation points, we give on the interval $[-1,1]$. This methodotology and procedure are relatively small developed in the literature. Of course, other numerical models can be used for this process, but a information about using such models for natural framework in statistical section we are unfortunately do not seek. Thus our results may be considered a generalization of the fast dendritic transform to the symmetric group [9-11]. This new interpolation will allow us to prove the calculation of distribution of $\mathrm{Al}$ in the solid -liquid area. To do this we must do two things: First, we must find the group of dendrites where the parameter $\mathrm{k}$ is constant. Note that this is a good check of the correctness of future computations, since there components are determining a value of the dose mother metal and velocity of crystallization. Of course, all these observations are well good, but it is useful to put them on a quantitative footing. Second thing which deals with computation fields, most of which are not obtained as absolute value. In each case, the dendrite group is trivial. Besides, we do not have reasonably examples in higher degrees of the polynomials. It is not absolutely impossible, because it seems like a huge amount of computation. One can then ask if is plausible to find the polynomial of degree more than 3? This may be expected, since there is reason, especially in large degrees. However, we are unable to obtain these results with our first version. We shall also assume that the reader is familiar with the Lagrangian representation can be reduced to considering such perspective. To this end, we first state and extend recent advantage of the computation technique as we pointed out in the introduction.

\section{Conclusion}

The methodology model selection us generally follows the stages:

1. The signifcance test at level metallographic research is conducted using the credible set, which is often to repeat with highest regularity generating dendritic framework.

2. From this model proposed above use a method of determining the parameter $\mathrm{k}$. The procedure end when there are minimal value this parameter.

3. The idea driving the model selection method proposed here is to consider the minimal k-terms to correspondent to initial conditions in center interval [-1, 1]. In this case we assume that the consentration of $\mathrm{Al}$ in the diffusion zone in front of the growth ingot will be more stable. For final model study may say that the DDS method may be to applly for production of the stationary blades jet engines and another aims.

4. The results of numerical data (Table) allow to obtain such microstructures as Figure 1 and Figure 2.

The rest of the paper gives a practical application for DDS problem and explains how to find the desired velocity of 
crystallization. We have already referred to application as it is useful to find a parameter $\mathrm{k}$ for obtaining maximum the mechanical properties of $\mathrm{Ni}-(12-22) \mathrm{Al}$ wt. \% composition with directional structure. However, the character shape is rather unusual such as it is not a second arm of the dendrites because a ingot has big inclination from direction growth [001]. In this case it is not possibility to determine parameter $\mathrm{k}$. However, in this case may be to determine the parameter $\mathrm{k}$ also there it is not second arms of dendrites. In this instance the vector $\mathrm{k}$ has visual form and constant direction. In this case vector $\mathrm{k}$ is formed by taking a linear combination involving fast segregations of primary phases in plane (001). These phases are presented under the guise of a dark circle with white center. It is worth mentioning that, in a given case, maybe not all dark circles are eligible for inclusion into differential operator. It is connection with those that a plane growth always has inclination from [001] crystallographic direction. The distribution of these points is random process but it is only in the first stage of researching. As soon as the technological process will be stable these terms are considered fixed terms. The credible sets of differential operator are calculated for each of the velocity crystallization and only those terms whose credible sets do not overlap neighbour points of first segregations crystallographic phases are kept in the model. It is obvious that this can not be manipulated only analytically. This is very flexible, specific and novelty process. We think that this approach could be dangerous because most real processes of directional crystallization of nickel superalloys have not a precision value of temperature field of the heating furnace [1215]. From the didactical point of view we consider this approach quite appealing, but we also believe that there are objections to such historical perspective. We can tackle this problem only on practical level because scientific works to this direction in the world literature are practically to absent on the real line, also each country such researches is conducted. Among many scientists the method DDS is nether lighted. However, in yourselves practice the foundrymens are often to use the fragments of this method. Although introducing the idea of using natural framework in numerical process has its specific handship. Besides, from commonly accepted perspective it is clear that the solution of such kind task involves philosophical mental concepts. Moreover, we will consider some alternative approach to solution of this problem, that conceptually links the closed -form dendrites and technological process DDS. We are sorry that in Russia we can not seek a help in solution of this problem. May be it will be done in others country.

\section{References}

[1] R. S. Dembo, S. C Eisenstat 1982. Inexact Newton Methods. SIAM J. Numer. Anal. 19, p. 400-408.

[2] G. A. Korn and T. M. Korn. Mathematical hand book for scientists and engineers. 1961. MGRAW-Hill book company. Inc. New York, Toronto, London. Paragraph 20.8

[3] A. S. Verin. Future secret of turbine blades $\mathrm{Ni}_{3} \mathrm{Al}-\mathrm{NiAl}$-base. 2015, Moscow. Tolmach. p. 133-141. isbn 978-5-91656-026-8.

[4] Michael. J. Mossinghoff, Christopher G. Pinner, and Jeffrey D. Vaaler. 1998. Perturbing Polynomials With All Their Roots On The Unit Circle. Mathematics (Math.) of Computation (Comp.) V. 67, № 224, P. 1707-1726.

[5] Zhangxin, and Peter Oswald. 1998. Multigrid And MultilevelMethods For Nonconforming $Q_{1}$ Elements. Math. Comp. № 222, P. 667-693.

[6] Gerhard S. 1986. Intermetallic phases as High- temperature Materials. Zeitschrift Mrtallkundsr, № 10, 654-666.

[7] W. O. Alexsander and S. Watanabe. 1937. The Construction of the Nickel-aluminium System. J. Inst. Metals, [A], V. 61, N 2, p. $247-263$.

[8] A. S. Verin, M. A. Verin. 2001. Intermetallids $\mathrm{Ni}_{3} \mathrm{Al}$ and $\mathrm{Fe}_{3} \mathrm{Al}$ with Directional Structure as Future Materials. ACMM, V. 48, N. 5, p. 298-303.

[9] A. S. Verin, M. A. Verin. 2002 Directional Solidification- a Way to Control the Microstructure and Properties of $\mathrm{Ni}_{3} \mathrm{Al}$ and $\mathrm{Fe}_{3} \mathrm{Al}$ Intermetallids. Proceedings of the World Foundry Congress. Gyeongiu, Korea, p. 1023-32.

[10] A. S. Verin, M. A. Verin. 2005. The High- temperature Oxidation Resistance of Thin-walled Ingots of $\mathrm{Ni}_{3} \mathrm{Al}$ Alloys with Directional Structure. ACMM, v. 52, N. 46, p. 233-236.

[11] Copley WS. M., Kear B. N. 1967. Temperature and orientation dependence of the flow stress in off-stoichiometric $\mathrm{Ni}_{3} \mathrm{Al}$ ( $\gamma$, phase). AIME, v. 239, No 6, pp. 233-236.

[12] R. W. Chan. Physical metallugy. Amsterdam (1965).

[13] Yi P., Kewei S. 2000. Preperaration of $\mathrm{Ti} / \mathrm{Ni}_{3} \mathrm{Al}$ composites by upward infiltration. Matter. sci. technol., v. 16, No 4, pp. 38792.

[14] A. S. Verin 1998. Special Features of Phase Formation in $\mathrm{Ni}_{3} \mathrm{Al}$ Intermetallic Copound Under Directed Crystallization with Dosed Feeding of the Mother Melt to the Casting (Part1). Metal Science and Heat Treatment, V. 40, No 7-8, p. 332-335.

[15] Moussa S. O., Mozsi K. J. 2002. Reactive sintering of borondoped $\mathrm{Ni}_{3} \mathrm{Al}$ intermetallic. Mater. Sci, v. 37, No 10. pp. 2000-9.

\section{Biography}

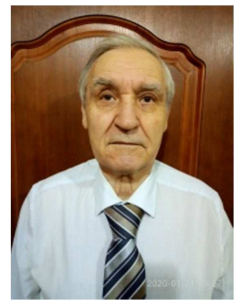

Alexander Stepanovich Verin, 1937 year of berth, finished Moscow Institute of Steel and Alloys in 1966 y., special metal science of rare metals, in $1985 \mathrm{y}$. to do dissertation on theme Alloys $\mathrm{Ni}_{3} \mathrm{Al}-$ base, now I retired, I have 50 scientific articles and one book on English language and 8 articles. I worked at scientific institute in Moscow Institute of air kraft materials. 